\title{
Prediction of Intraoperative Trifecta Achievement during Laparoscopic Partial Nephrectomy
}

\author{
Ovidiu-Spiru Barnoiu ${ }^{*}{ }^{(D}$, F. J. Baron${ }^{2}$, T. Sæter ${ }^{3}$, A. 0. Tysland ${ }^{1}$, A. Andersen ${ }^{4}$ \\ ${ }^{1}$ Department of Urology, Sorlandet Hospital, Kristiansand, Norway \\ ${ }^{2}$ Department of Biostatistics, University of Malaga, Malaga, Spain \\ ${ }^{3}$ Department of Clinical and Molecular Medicine, Norwegian University of Science and Technology, Trondheim, Norway \\ ${ }^{4}$ Department of Urology, Sorlandet Hospital, Arendal, Norway \\ Email: *ovidiu.spiru.barnoiu@gmail.com
}

How to cite this paper: Barnoiu, O.-S., Baron, F.J., Sæter, T., Tysland, A.O. and Andersen, A. (2021) Prediction of Intraoperative Trifecta Achievement during Laparoscopic Partial Nephrectomy. Open Journal of Urology, 11, 6-16.

https://doi.org/10.4236/oju.2021.111002

Received: December 27, 2020

Accepted: January 24, 2021

Published: January 27, 2021

Copyright $\odot 2021$ by author(s) and Scientific Research Publishing Inc. This work is licensed under the Creative Commons Attribution International License (CC BY 4.0).

http://creativecommons.org/licenses/by/4.0/

cC) (i) Open Access

\begin{abstract}
Purpose: We introduce the concept of intraoperative Trifecta during laparoscopic partial nephrectomy (LPN) as the simultaneous achievement of estimated blood loss (EBL) $<500 \mathrm{ml}$, warm ischemia time (WIT) $<20$ minutes and minimal changes of the intraoperative course. The study's aim was to find preoperative factors that could predict the likelihood of achieving intraoperative Trifecta and build a surgical nomogram. Methods: We retrospectively evaluated 122 patients who underwent LPN. Preoperative factors like age, sex, body-mass index (BMI), kidney function, tumor characteristics (R.E.N.A.L. score) and Charlson-Comorbidity-Index (CCI) were recorded. Intraoperative complication (IOC) was graded according to the Rosenthal classification. $\mathrm{R}$ software was used to find a predicting model for achievement of Trifecta using preoperative variables and a nomogram was built. Results: The surgical features include median EBL of $100 \mathrm{ml}$ having $6.5 \%$ bleed $>500$ $\mathrm{ml}$, median WIT of 12 minutes having $7.3 \%$ more than 20 minutes. There was recorded a $12.3 \%$ IOC with a mean Rosenthal's grade of 0.2 . Intraoperative Trifecta was achieved in 105 patients (86\%) and three preoperative factors were chosen for the predictive model: BMI $(\mathrm{p}=0.041)$, CCI $(\mathrm{p}=0.037)$ and RENAL score $(\mathrm{p}=0.002)$. A nomogram was generated and the ROC-AUC of the model was $75.8 \%$. Conclusion: We have defined an intraoperative Trifecta concept as the achievement of EBL $<500 \mathrm{ml}$, WIT $<20$ minutes and minimal changes of the intraoperative course. A nomogram was developed from preoperative factors like BMI, CCI and R.E.N.A.L. score. It can be used to estimate the probability of Trifecta achievement in patients treated with LPN.
\end{abstract}




\section{Keywords}

Intraoperative Complications, Laparoscopic Partial Nephrectomy, Prediction, Trifecta

\section{Introduction}

Partial nephrectomy (PN) represents the standard of care for patients diagnosed with T1a kidney cancer [1]. Minimally invasive nephron-sparing surgery should be performed if this approach does not compromise oncological, functional and perioperative outcomes. However, these approaches are technically challenging and are associated with a high rate of complications that has been reported in up to $30 \%$ of cases [2]. Hemorrhage and transient renal insufficiency are the most common concerns during PN. An increased hospital mortality [3] and risk for intraoperative transfusion [4] were found in those patients whose estimated blood loss (EBL) exceeded $500 \mathrm{ml}$. Vascular clamping during PN is associated with kidney function impairment and attempts should be done to limit warm ischemia time (WIT) to 20 minutes [5].

Many trials fail to report intraoperative complications (IOC). Rosenthal et al. defined in 2015 and classified IOC depending on the need for treatment and the severity of complication [6]. We tried to evaluate these complications by using a Trifecta concept and we defined the intraoperative Trifecta as the achievement of EBL $<500 \mathrm{ml}$, WIT $<20$ minutes and no other change of normal intraoperative course/or changes without any consequences.

The study's aim was to find preoperative factor that could predict the likelihood of achieving intraoperative Trifecta and build a surgical nomogram.

\section{Materials and Methods}

We retrospectively evaluated the patients who underwent laparoscopic PN (LPN) at our institution between January 2015-December 2018 and 122 patients had registered the IOC directly after surgery and qualified for the statistical analysis.

In order to achieve our study's aim, we first did a univariate analysis of preoperative variables and a multivariate one, having achievement of Trifecta as the main variable, and then built a nomogram for those factors that showed to be predictable for our newly introduced intraoperative Trifecta.

We analyzed the preoperative factors like age, sex, body-mass index (BMI), kidney function evaluated by estimated glomerular filtration rate (eGFR) and tumor characteristics, like size, side, type (solid or cystic) and number. Morphometric score like R.E.N.A.L.-score (Radius, Exophytic/endophytic, Nearness, Anterior/posterior, Location), as described by Kutikov [7], was assigned in an unblinded manner by the same urologist (OSB). Comorbidity status was evaluated using the Charlson Comorbidity Index (CCI) and physical status by the American Society of Anesthesiologists (ASA) classification system. All the data 
were collected from patients' electronic medical record, in a retrospective manner, and stored in our kidney cancer database reviewed and accepted by our Institution's Research and Ethics Committee. Variables like BMI were obtained using an online calculator

(https://www.cdc.gov/healthyweight/assessing/bmi/adult_bmi/english_bmi_calc ulator/bmi_calculator.html) the same way as CCI

(https://www.mdcalc.com/charlson-comorbidity-index-cci). GFR was automatically calculated by our Laboratory Unit.

There were to surgeons that performed the procedures and both of them have overcome a learning curve of more than 300 LPN before the observational period. Both surgeons started doing LPN in our Unit in 2004 and performed more than 30 procedures per year, each. The procedures were performed using both pure laparoscopic or hand-assisted technique.

The intraoperative covariates consisted of total operation time, WIT and EBL, both recorded and agreed between the surgeon and the anesthesiologist, the usage of drainage tube, and performance of standard or hand-assisted technique.

Any deviation from the ideal intraoperative course occurring between skin incision and skin closure, regardless whether it was related to surgery or anesthesia, was considered as an IOC and graded according to the Rosenthal classification [6]. The classification includes four grades depending on the need for treatment (no need, grade 1; need for treatment, grade 2) and the severity of the complication (life-threatening/permanent disability, grade 3; death, grade 4). Because of expected variability in reporting complications of grade 1 , both grade 0 (no deviation from the ideal intraoperative course) and 1 (any deviation without need of treatment) war taken together for subsequent analysis. No cystic lesion were cut or ruptured during the resection.

\section{Statistical Analysis}

Demographic and clinical outcomes were analyzed using descriptive statistics. $\mathrm{R}$ Core Team (2019) software was used to find the best predicting model for the achievement of intraoperative Trifecta using preoperative variables. A nomogram was built and receiver operating curve (ROC) and areas under the curve (AUC) were calculated and used to quantify predictive discrimination. Statistical significance was set at $\mathrm{p}<0.05$. Internal validation and variance of AUC-ROC processes were performed using bootstrapping with 10,000 repetitions.

\section{Results}

The clinical characteristics of the population are summarized in Table 1. The cohort comprised $65.6 \%$ males, mean BMI was $27.5 \mathrm{~kg} / \mathrm{m}^{2}$ and median CCI was 5. Most of the patients had ASA II with a median preoperative kidney function of $89.5 \mathrm{ml} / \mathrm{min} / 1.73 \mathrm{~m}^{2}$. Most of the tumors were solid (73.8\%) with a median tumor diameter of $2.45 \mathrm{~cm}$ and a median RENAL score of 6.5 points. The surgical features include a median operation time of 157 minutes, median EBL 100 
$\mathrm{ml}$ having 6.5\% (eight patients) bleed more than $500 \mathrm{ml}$, median WIT of 12 minutes having $7.3 \%$ (nine patients) more than 20 minutes. There was used a drainage tube in $82 \%$ of the patients and a hand-assisted technique was performed in $23 \%$. General and surgical postoperative complications (POC) occurred in $27 \%$ and $4 \%$ respectively (three patients with bleeding, one bowel injury and one chylous ascites).

There was recorded a $12.3 \%$ ( 15 patients) IOC with a mean Rosenthal's grade of 0.213 . Of these 15 patients, six had a grade 1 , six had grade 2 and three had grade 3 (two conversions to nephrectomy and one splenectomy). Some patients (three) had more than one complication but only the highest grade was assigned.

Table 1. Patient demographics.

\begin{tabular}{|c|c|}
\hline & Overall $(n=122)$ \\
\hline \multicolumn{2}{|l|}{ Age } \\
\hline Mean (SD) & $61.5(12.2)$ \\
\hline Median [Min, Max] & $65.0[18.0,85.0]$ \\
\hline \multicolumn{2}{|l|}{ Sex } \\
\hline Man & $80(65.6 \%)$ \\
\hline Woman & $42(34.4 \%)$ \\
\hline \multicolumn{2}{|l|}{ BMI $\left(\mathrm{kg} / \mathrm{m}^{2}\right)$} \\
\hline Mean (SD) & $27.5(4.78)$ \\
\hline Median [Min, Max] & $27.1[17.3,47.9]$ \\
\hline \multicolumn{2}{|l|}{$\mathrm{CCI}$} \\
\hline Mean (SD) & $4.58(1.55)$ \\
\hline \multicolumn{2}{|l|}{ ASA } \\
\hline ASA I & $6(4.9 \%)$ \\
\hline ASA II & $71(58.2 \%)$ \\
\hline ASA III & $44(36.1 \%)$ \\
\hline ASA IV & $1(0.8 \%)$ \\
\hline \multicolumn{2}{|l|}{ Tumor type } \\
\hline Solid & $90(73.8 \%)$ \\
\hline Bosniak 2F & $0(0 \%)$ \\
\hline Bosniak 3 & $13(10.7 \%)$ \\
\hline Bosniak 4 & $19(15.6 \%)$ \\
\hline \multicolumn{2}{|l|}{ Tumor number } \\
\hline Mean (SD) & $1.04(0.237)$ \\
\hline \multicolumn{2}{|l|}{ Side } \\
\hline Right & $66(54.1 \%)$ \\
\hline Left & $56(45.9 \%)$ \\
\hline \multicolumn{2}{|l|}{ CT diameter } \\
\hline Mean (SD) & $2.69(1.20)$ \\
\hline \multicolumn{2}{|l|}{ R.E.N.A.L. score } \\
\hline Mean (SD) & $6.42(1.79)$ \\
\hline Median [Min, Max] & $6.50[4.00,10.0]$ \\
\hline
\end{tabular}

BMI: body mass index, CCI: Charlson Comorbidities Index, ASA: American Society of Anesthesiologists classification system. 
Intraoperative Trifecta was achieved in 105 patients (86\%) and four preoperative factors correlated with Trifecta: BMI ( $p=0.041)$, CCI $(p=0.037)$, CT diameter $(\mathrm{p}=0.036)$ and R.E.N.A.L. score $(\mathrm{p}=0.002)$ as shown in Table 2 . The best nomogram generated of $\mathrm{R}$ software included just BMI, CCI and R.E.N.A.L.-score (Figure 1). The ROC AUC of the model was 75.8\%. After a bootstrapping with 10,000 repetitions, the model reported a bias of -0.022 and a standard error of 0.216 .

Table 2. Multivariate analysis assessing the association between intraoperative Trifecta achievement and preoperative factors.

\begin{tabular}{|c|c|c|c|}
\hline & $\begin{array}{l}\text { Trifecta NOT achieved } \\
\qquad(\mathrm{n}=17)\end{array}$ & $\begin{array}{l}\text { Trifecta achieved } \\
\qquad(\mathrm{n}=105)\end{array}$ & $\mathrm{p}$-value \\
\hline \multicolumn{4}{|l|}{ Age } \\
\hline Mean (SD) & $63.9(11.2)$ & $61.1(12.4)$ & 0.348 \\
\hline \multicolumn{4}{|l|}{ Sex } \\
\hline Man & $14(82.4 \%)$ & $66(62.9 \%)$ & 0.196 \\
\hline Woman & $3(17.6 \%)$ & $39(37.1 \%)$ & \\
\hline \multicolumn{4}{|l|}{ BMI $\left(\mathrm{kg} / \mathrm{m}^{2}\right)$} \\
\hline Mean (SD) & $29.0(3.70)$ & $27.3(4.90)$ & 0.041 \\
\hline \multicolumn{4}{|l|}{$\mathrm{CCI}$} \\
\hline Mean (SD) & $5.29(1.86)$ & $4.47(1.47)$ & 0.037 \\
\hline \multicolumn{4}{|l|}{ Number } \\
\hline Mean (SD) & $1.06(0.243)$ & $1.04(0.237)$ & 0.746 \\
\hline \multicolumn{4}{|l|}{ Side } \\
\hline Right & $9(52.9 \%)$ & $57(54.3 \%)$ & 0.912 \\
\hline Left & $8(47.1 \%)$ & $48(45.7 \%)$ & \\
\hline \multicolumn{4}{|l|}{ CT diameter } \\
\hline Mean (SD) & 3.55 (1.77) & $2.56(1.02)$ & 0.036 \\
\hline \multicolumn{4}{|c|}{ R.E.N.A.L. score } \\
\hline Mean (SD) & $7.59(1.46)$ & $6.23(1.77)$ & 0.002 \\
\hline \multicolumn{4}{|l|}{ Surgeon } \\
\hline 1 & $14(82.4 \%)$ & $75(71.4 \%)$ & 0.518 \\
\hline 2 & $3(17.6 \%)$ & $30(28.6 \%)$ & \\
\hline \multicolumn{4}{|l|}{ Drainage } \\
\hline No & $0(0 \%)$ & $22(21.0 \%)$ & 0.081 \\
\hline Yes & $17(100 \%)$ & $83(79.0 \%)$ & \\
\hline \multicolumn{4}{|c|}{ Hand-assisted } \\
\hline Yes & $3(17.6 \%)$ & $25(23.8 \%)$ & 0.803 \\
\hline No & $14(82.4 \%)$ & $80(76.2 \%)$ & \\
\hline \multicolumn{4}{|l|}{ Tumor (Solid) } \\
\hline FALSE & $4(23.5 \%)$ & $28(26.7 \%)$ & 1 \\
\hline TRUE & $13(76.5 \%)$ & $77(73.3 \%)$ & \\
\hline
\end{tabular}

BMI: body mass index, CCI: Charlson Comorbidities Index, ASA: American Society of Anesthesiologists classification system. 


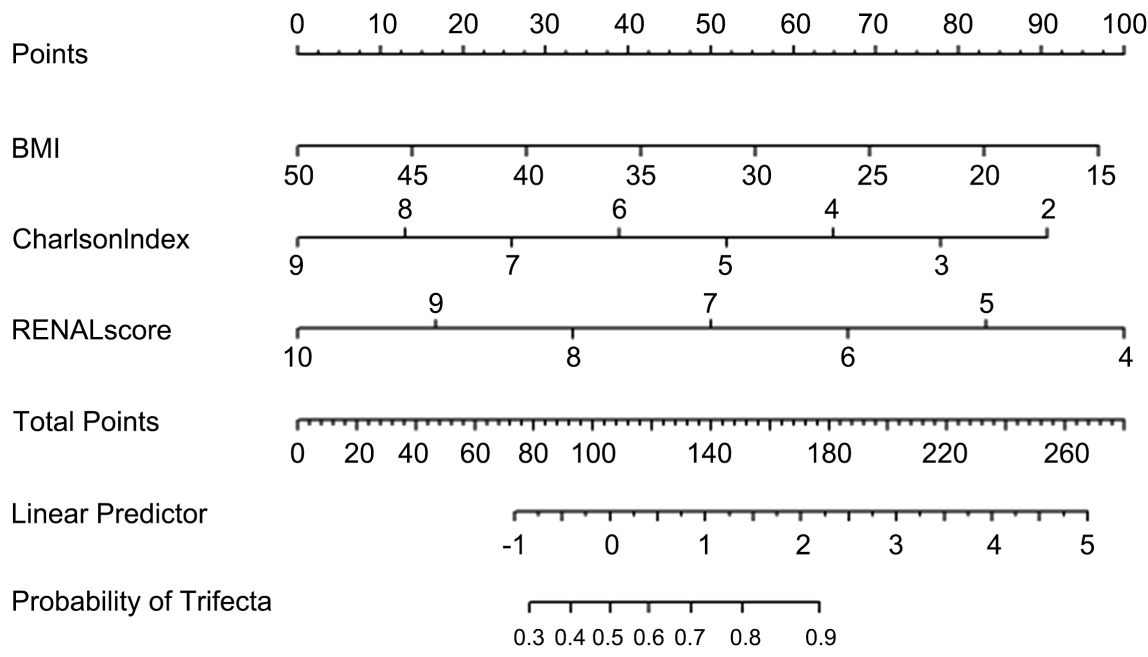

Figure 1. Nomogram predicting intraoperative Trifecta achievement. BMI: body mass index, CCI: Charlson Comorbidities Index.

\section{Discussion}

Various trifecta combinations exist, all of which are mostly used to measure postoperative outcomes after PN; for example Buffi [8] and Porpiglia [9] using the term MIC (negative Margin, Ischemia time $<20$ minutes and no major Complications), Khalifeh [10] encompassed no positive surgical margin, zero complications and WIT $<25$ minutes. Hung [11] defined Trifecta as a composite outcome of negative margin, no urological complications and no renal function loss of $>90 \%$. We are the first to define a Trifecta only using intraoperative variables and tried to correlate it with preoperative factors in order to predict its accomplishment. Our intraoperative Trifecta was achieved in $86 \%$ of the patients attesting to the efficacy of LPN in the minimally invasive surgical treatment of kidney cancer.

EBL is the first component in our definition and intraoperative hemorrhage from the PN bed is an important concern. In no case in our group did significant hemorrhage occur during parenchymal and tumor resection, and in just two cases critical bleeding occurred after hilar unclamping. An increased risk for intraoperative transfusion [4] was found in those patients whose EBL exceeded $500 \mathrm{ml}$, the value chosen for our Trifecta. Most of the patients bleed $<100 \mathrm{ml}$ and only eight bleed $>500 \mathrm{ml}$ with need of transfusion in five patients (three of them with grade 2 according with Rosenthal classification and the 2 patients that needed nephrectomy and intraoperative transfusion).

Our second component was WIT with the cut-off value of 20 minutes as first used by Thompson et al. [5]. Zargar [12] and Khalifeh [10] used the 25 minutes cut-off to define their Trifecta and Propiglia [13] have shown no renal function impairment in WIT < 30 minutes. Ficarra [14] reported a group of patients with $36 \%$ having a WIT over 20 minutes and found tumor size, PADUA score and surgeon experience to be predictors of a WIT more than 20 minutes and IOC. In our study median WIT was 12 minutes and only in $7.3 \%$ was more than $20 \mathrm{mi}$ - 
nutes and in two patients more than 30 minutes, which, again, suggests that LPN is a feasible technique.

Third, and last component, included in our Trifecta was the common variableused in all previous definitions being that the presence of complications, in our case intraoperative complications. The classification most widely used and with most evidence in literature we found to be the Rosenthal classification [6]. Grade 0 and 1 were taken together as both no complication (grade 0 ) and complication with no need for treatment (grade 1), have no consequence for the surgical outcome. The six patients assigned as grade 1 included four patients with bleeding from trocar site, kidney dissection or kidney vein injury without any need for transfusion and two patients with respiratory distress or atrial flutter recorded intraoperatively without any hemodynamic consequence or need for treatment. Only nine patients had complications grade 2 and 3 and no death (grade 4) was recorded in our series.

BMI, CCI and R.E.N.A.L.-score were the preoperative factors that were predictive for achievement of intraoperative Trifecta. It is worldwide believed that high BMI can increase the operation time and blood loss but data regarding association between elevated BMI and POC after LPN is controversial. Wiens [15] showed that obese patients undergoing LPN are not at significantly increased risk of complication relative to non-obese patients and that comorbidity status and R.E.N.A.L.-score should be the main criteria to take into account to evaluate feasibility for LPN. On the contrary, Kott [16] found that a BMI over $30 \mathrm{~kg} / \mathrm{m}^{2}$ was a significant factor for POC associated with robot assisted LPN. In our analysis there was a relative significant correlation between achievement of intraoperative Trifecta and BMI. Comorbidity status, assessed with CCI, was a predictive factor for our Trifecta, and there are authors like Larcher [17] showing a correlation between CCI and complications after LPN. As for patients performance status we included also ASA score that failed to reach significance at multivariable analysis. ASA score was found to be significant predictive factor in a nomogram used by Mari [18] in the RECORD2 project in order to predict the likelihood of POC. Tumor anatomy is also a well-known factor that correlates with POC and anatomical characteristics of the renal tumor could be evaluated by many morphometric scores like, for example, the R.E.N.A.L.-score by Kutikov [7], PADUA by Ficarra [19] and C-index by Simmons [20]. Other anatomical characteristics like renal tumor invasion [21] were not assessed by the RENAL score that we used in our study and chosen as the standard morphometric score in our unit because of its worldwide demonstrated reproducibility and validity [22] [23]. This was the preoperative factor that most significantly correlated with the intraoperative Trifecta. We did not find a correlation between the suffix of the R.E.N.A.L.-score and the Trifecta as seen by Reddy [24]. There are few reports evaluating the true value of a single component included in the anatomical scores [25] and, in our analysis, the tumor density, side and number were not significant variables while tumor diameter was. 
Other preoperative factors like age, sex, and surgeon, use of drainage tube or use of hand-assisted technique did not correlate with intraoperative Trifecta achievement. Age is a factor found to predict POC by two main nomograms of Larcher [17] and Mari [18]. Median age for these studies was 73 and 64 years respectively, compared to a younger population in our series with median age of 61 years. Age is a factor included in the CCI that correlated better in our study than age alone. Bindayi and RESURGE group [26] analyzed the Trifecta outcomes in elderly patients over 75 years and found a $40 \%$ Trifecta achievement and less transfusion and lower intraoperative complications in the Trifecta patients.

In our group, no difference was seen between the two surgeons as both have overcome alearning curve of more than 300 procedures, being 70 procedures needed according to Buffi to achieve Trifecta in $87.9 \%$ of the patients [8]. No difference between hand-assisted and pure laparoscopic technique was seen in order to achieve all three components of our Trifecta. Azawi [27] studied the impact of using a hand-assisted technique on the learning curve and found that the surgeon must perform 40 procedures to obtain a WIT of five minutes. Our study did not individually analyze the correlation between hand-assisted laparoscopic PN (HALPN) and R.E.N.A.L.-score alone but the three factors of Trifecta together. Elsamra [28] compared HALPN with the robot-assisted and found no significant advantage of robot-assisted over HALPN in short-term outcomes.

Our study is the first, in our knowledge, evaluating the factors that could predict IOC alone, and building a nomogram out of preoperative risk factors. A predictive tool, such as this, can enable clinicians to evaluate the risk of IOC according to specific patient and tumor related factors. It can also estimate more accurately the risk stratification on each individual case before treatment and could guide the learning curve of future kidney cancer surgeons allowing to choose the right patient for the right step in the learning curve.

The limitations of the study are the relative small sample size. The tumors operated on were relatively small and low to intermediate complexity, which we believe represents patients we typically treat in our daily practice in this era of CT-diagnosed abdominal symptoms and incidental finding of early kidney cancer. The study offers several opportunities for future research by using the intraoperative Trifecta concept in the robot-assisted field and in bigger national kidney cancer registers. It can be also used to analyze the correlation with postoperative outcomes and thereby measure the effect of its achievement.

The nomogram from our study should to be tested on multicenter cohorts in order to externally validate and generalize our findings. We believe there was a high-quality report of IOC that was guaranteed by the rigorous recording of data of both surgeon and anesthesiologist in our complications register.

\section{Conclusion}

We have defined an intraoperative Trifecta concept to evaluate the IOC during 
LPN as the achievement of EBL $<500 \mathrm{ml}$, WIT $<20$ minutes and no other changes or changes without any consequences over the normal intraoperative course. A nomogram was developed from preoperative predictive factors including BMI, CCI and R.E.N.A.L.-score and it can be used to estimate the probability of intraoperative Trifecta achievement in patients treated with LPN.

\section{Acknowledgements}

The study was approved by the local ethics committee. All patients signed informed consent.

\section{Conflicts of Interest}

The authors report no conflicts of interest and no funding for the study.

\section{References}

[1] Ljungberg, B., Albiges, L., Abu-Ghanem, Y., et al. (2019) European Association of Urology Guidelines on Renal Cell Carcinoma: The 2019 Update. European Urology, 75, 799-810. https://doi.org/10.1016/j.eururo.2019.02.011

[2] Mari, A., Antonelli, A., Bertolo, R., et al. (2017) Predictive Factors of Overall and Major Postoperative Complications after Partial Nephrectomy: Results from a Multicenter Prospective Study (The RECORD 1 Project). European Journal of Surgical Oncology, 43, 823-830. https://doi.org/10.1016/j.ejso.2016.10.016

[3] Carson, J.L., Spence, R.K., Poses, R.M., et al. (1988) Severity of Anemia and Operative Mortality and Morbidity. The Lancet, 1, 727-729.

https://doi.org/10.1016/S0140-6736(88)91536-X

[4] Wu, W.C., Smith, T.S., Henderson, W.G., et al. (2010) Operative Blood Loss, Blood Transfusion, and 30-Day Mortality in Older Patients after Major Noncardiac Surgery. Annals of Surgery, 252, 11-17. https://doi.org/10.1097/SLA.0b013e3181e3e43f

[5] Thompson, R.H., Frank, I., Lohse, C.M., et al. (2007) The Impact of Ischemia Time during Open Nephron Sparing Surgery on Solitary Kidneys: A Multi-Institutional Study. The Journal of Urology, 177, 471-476. https://doi.org/10.1016/j.juro.2006.09.036

[6] Rosenthal, R., Hoffmann, H., Clavien, P.A., et al. (2015) Definition and Classification of Intraoperative Complications (CLASSIC): Delphi Study and Pilot Evaluation. World Journal of Surgery, 39, 1663-1671. https://doi.org/10.1007/s00268-015-3003-y

[7] Kutikov, A. and Uzzo, R.G. (2009) The RENAL Nephrometry Score: A Comprehensive Standardized System for Quantitating Renal Tumor Size, Location and Depth. Journal of Urology, 182, 844-853. https://doi.org/10.1016/j.juro.2009.05.035

[8] Buffi, N., Lista, G., Larcher, A., et al. (2012) Margin, Ischemia, and Complications (MIC) Score in Partial Nephrectomy: A New System for Evaluating Achievement of Optimal Outcomes in Nephron-Sparing Surgery. European Urology, 62, 617-618. https://doi.org/10.1016/j.eururo.2012.06.001

[9] Porpiglia, F., Bertolo, R., Amparore, D., et al. (2013) Margins, Ischaemia and Complications Rate after Laparoscopic Partial Nephrectomy: Impact of Learning Curve and Tumor Anatomical Characteristics. BJU International, 112, 1125-1132. https://doi.org/10.1111/bju.12317

[10] Khalifeh, A., Autorino, R., Eyraud, R., et al. (2013) Three-Year Oncologic and Renal 
Functional Outcomes after Robot-Assisted Partial Nephrectomy. European Urology, 64, 744-750. https://doi.org/10.1016/j.eururo.2013.03.052

[11] Hung, A.J., Cai, J., Simmons, M.N., et al. (2013) "Trifecta" in Partial Nephrectomy. Journal of Urology, 189, 36-42. https://doi.org/10.1016/j.juro.2012.09.042

[12] Zargar, H., Allaf, M.E., Bhayani, S., et al. (2015) Trifecta and Optimal Perioperative Outcomes of Robotic and Laparoscopic Partial Nephrectomy in Surgical Treatment of Small Renal Masses: A Multi-Institutional Study. BJU International, 116, 407-414. https://doi.org/10.1111/bju.12933

[13] Porpiglia, F., Renard, J., Billia, M., et al. (2007) Is Warm Ischemia over 30 Minutes during Laparoscopic Partial Nephrectomy Possible? One-Year Results of a Prospective Study. European Urology, 52, 1170-1178. https://doi.org/10.1016/j.eururo.2007.04.024

[14] Ficarra, V., Bhayani, S., Porter, J., et al. (2012) Predictors for Warm Ischemia Time and Perioperative Complications in a Multicenter, International Series of Robot-Assisted Partial Nephrectomy. European Urology, 61, 395-402. https://doi.org/10.1016/j.eururo.2011.10.046

[15] Evan, J.W., Deepak, K.P., Ruchi, C., et al. (2017) Feasibility of Laparoscopic Partial Nephrectomy in the Obese Patient and Assessment of Predictors of Perioperative Outcomes. Urology Annals, 9, 27-31. https://doi.org/10.4103/0974-7796.198888

[16] Kott, O., Golijanin, B. and Pereira, J. (2019) The BMI Paradox and Robotic Assisted Partial Nephrectomy. Frontiers in Surgery, 6, 74.

https://doi.org/10.3389/fsurg.2019.00074

[17] Larcher, A., Fossati, N., Tian, Z., et al. (2016) Prediction of Complications Following Partial Nephrectomy: Implications for Ablative Techniques Candidates. European Urology, 69, 676-682. https://doi.org/10.1016/j.eururo.2015.07.003

[18] Mari, A., Campi, R. and Schiavina, R. (2019) Nomogram for Predicting the Likelihood of Postoperative Surgical Complications in Patients Treated with Partial Nephrectomy: A Prospective Multicenter Observational Study (the RECORD 2 Project). BJU International, 124, 93-102. https://doi.org/10.1111/bju.14680

[19] Ficarra, V., Novara, G., Secco, S., et al. (2009) Preoperative Aspects and Dimensions Used for an Anatomical (PADUA) Classification of Renal Tumors in Patients Who Are Candidates for Nephron-Sparing Surgery. European Urology, 56, 785-793. https://doi.org/10.1016/j.eururo.2009.07.040

[20] Simmons, M.N., Ching, C.B., Samplaski, M.K., et al. (2010) Kidney Tumor Location Measurement Using the C-Index Method. Journal of Urology, 183, 1708-1713. https://doi.org/10.1016/j.juro.2010.01.005

[21] Nisen, H., Ruutu, M., Glucker, E., et al. (2014) Renal Tumor Invasion Index as a Novel Anatomical Classification Predicting Urological Complications after Partial Nephrectomy. Scandinavian Journal of Urology, 48, 41-51. https://doi.org/10.3109/21681805.2013.797491

[22] Matos, A.C., Oglio, M.F.D., Colombo, J.R., et al. (2017) Predicting Outcomes in Partial Nephrectomy: Is the Renal Score Useful? International Brazilian Journal of Urology, 43, 422-431. https://doi.org/10.1590/s1677-5538.ibju.2016.0315

[23] Liu, Z.W., Olweny, E.O., Yin, G., et al. (2013) Prediction of Perioperative Outcomes Following Minimally in Asive Partial Nephrectomy: Role of the RENAL Nephrometry Score. World Journal of Urology, 31, 1183-1189.

https://doi.org/10.1007/s00345-012-0876-3

[24] Reddy, U.D., Pillai, R. and Parker, R.A. (2014) Prediction of Complications after Partial Nephrectomy by RENAL Nephrometry Score. Annals of the Royal College 
of Surgeons of England, 96, 475-479.

https://doi.org/10.1308/003588414X13946184903522

[25] Volpe, A. and Terrone, C. (2011) Anatomic Classification Systems of Renal Tumors: New, Useful Tools in Real Surgical Oncology. European Urology, 60, 731-733. https://doi.org/10.1016/j.eururo.2011.07.038

[26] Bindayai, A., Autorino, R., Capitanio, U., et al. (2019) Trifecta Outcomes of Partial Nephrectomy in Patients over 75 Year Old: Analysis of the Renal Surgery in Elderly (Resurge) Group. European Urology Focus, pii: S2405-4569(19)30030-6.

[27] Azawi, N.H., Norus, T.P., Wittendorff, H.E., et al. (2014) Hand-Assisted Partial Nephrectomy with Early Arterial Clamp Removal: Impact of the Learning Curve. Scandinavian Journal of Urology, 48, 538-543.

https://doi.org/10.3109/21681805.2014.925499

[28] Elsamra, S.E., Leone, A.R., Lasser, M.S., et al. (2013) Hand-Assisted Laparoscopic versus Robot-Assisted Laparoscopic Partial Nephrectomy: Comparison of Short-Term Outcomes and Cost. Journal of Endourology, 27, 182-188.

https://doi.org/10.1089/end.2012.0210 